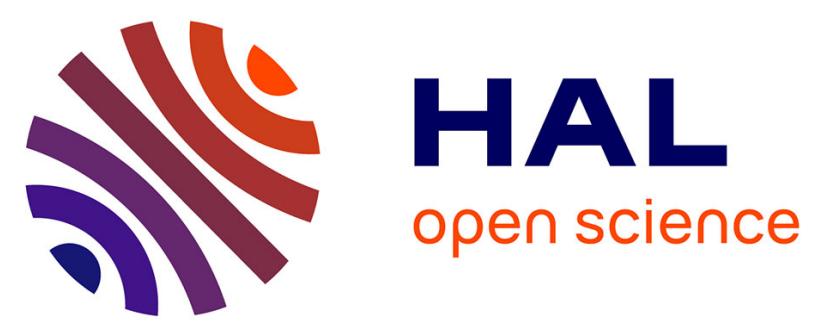

\title{
Disentangling normal aging from Alzheimer's disease in structural magnetic resonance images
}

Marco Lorenzi, Xavier Pennec, Giovanni B. Frisoni, Nicholas Ayache

\section{To cite this version:}

Marco Lorenzi, Xavier Pennec, Giovanni B. Frisoni, Nicholas Ayache. Disentangling normal aging from Alzheimer's disease in structural magnetic resonance images. Neurobiology of Aging, 2015, 36, pp.S42-S52. 10.1016/j.neurobiolaging.2014.07.046 . hal-01061017

\section{HAL Id: hal-01061017 https://hal.inria.fr/hal-01061017}

Submitted on 4 Sep 2014

HAL is a multi-disciplinary open access archive for the deposit and dissemination of scientific research documents, whether they are published or not. The documents may come from teaching and research institutions in France or abroad, or from public or private research centers.
L'archive ouverte pluridisciplinaire HAL, est destinée au dépôt et à la diffusion de documents scientifiques de niveau recherche, publiés ou non, émanant des établissements d'enseignement et de recherche français ou étrangers, des laboratoires publics ou privés. 


\title{
Disentangling Normal Aging from Alzheimer's Disease in Structural MR Images
}

\author{
Marco Lorenzi ${ }^{1}$, Xavier Pennec ${ }^{1}$, Giovanni Frisoni ${ }^{2,3}$, and Nicholas \\ Ayache $^{1}$, for the Alzheimer's Disease Neuroimaging Initiative* \\ ${ }^{1}$ Asclepios research Project, INRIA Sophia Antipolis, France. \\ ${ }^{2}$ IRCCS Fatebenefratelli, Brescia, Italy. \\ ${ }^{3}$ University Hospitals and University of Geneva, Geneva, \\ Switzerland.
}

\begin{abstract}
The morphology observed in the brains of patients affected by Alzheimer's disease $(\mathrm{AD})$ is a combination of different biological processes, such as normal aging and the pathological matter loss specific to AD. The ability to differentiate between these biological factors is fundamental in order to reliably evaluate pathological AD-related structural changes, especially on the earliest phase of the disease, at prodromal and pre-clinical stages. Here we propose a method based on non-linear image registration to estimate and analyze from observed brain morphologies the relative contributions

* Data used in preparation of this article were obtained from the Alzheimers Disease Neuroimaging Initiative (ADNI) database (adni.loni.usc.edu). As such, the investigators within the ADNI contributed to the design and implementation of ADNI and/or provided data but did not participate in analysis or writing of this report. A complete listing of ADNI investigators can be found at: http://adni.loni.usc.edu/wp-content/uploads/how_to_apply/ADNI_ Acknowledgement_List.pdf
\end{abstract}


from aging and pathology. In particular, we first define a longitudinal model of the brain's normal aging process from serial T1 MR scans of 65 healthy participants. The longitudinal model is then used as a reference for the cross-sectional analysis. Given a new brain image, we then estimate its anatomical age relative to the aging model; this is defined as a morphological age shift with respect to the average age of the healthy population at baseline. Finally, we define the specific morphological process as the remainder of the observed anatomy after the removal of the estimated normal aging process. Experimental results from 105 healthy participants, 110 subjects with mild cognitive impairment (MCI), 86 with MCI converted to $\mathrm{AD}$, and $134 \mathrm{AD}$ patients, provide a novel description of the anatomical changes observed across the AD time span: normal aging, normal aging at risk, conversion to MCI and the latest stages of AD. More advanced AD stages are associated with an increased morphological age shift in the brain and with strong disease-specific morphological changes affecting mainly ventricles, temporal poles, the entorhinal cortex and hippocampi. Our model shows that AD is characterized by localized disease-specific brain changes as well as by an accelerated global aging process. This method may thus represent a more precise instrument to identify potential clinical outcomes in clinical trials for disease modifying drugs.

\section{Introduction}

The objective of computational anatomy when applied to neurodegenerative diseases, such as Alzheimer's disease (AD), is to understand the pathological changes affecting brain morphology [Frisoni et al., 2010, Scahill et al., 2002]. However, the morphology of the brain affected by AD is not completely related to the disease, especially in asymptomatic and prodromal stages, since 
the brain structure is also the result of patient phenotype and clinical history. In a brain affected by $\mathrm{AD}$, we can identify two major processes contributing to morphological changes: normal aging and AD pathology itself.

- Age-related anatomical changes. It is known that aging is related to progressive impairment of neural mechanisms [Burke and Barnes, 2006], to chemical alterations in the brain and to changes in cognition and behaviour [Hof and Mobbs, 1984]. It has been observed that morphological changes in the aging brain are heterogeneous and primarily lead to grey matter loss in frontal, temporal and parietal areas [Sowell et al., 2003, Long et al., 2012].

- Disease-related anatomical changes. $\mathrm{AD}$ is a neurodegenerative disease characterized by the co-occurrence of different phenomena. It starts with the deposition of amyloid plaques and tau proteins in neurofibrillary tangles, which is followed by the development of function brain loss, and finally by widespread structural atrophy [Jack et al., 2010]. The typical pattern of brain tissue loss seen in AD mirrors tau deposition [Thompson et al., 2003] and involves primarily hippocampi, the enthorinal cortex, the posterior cingulate, and secondarily the temporal, parietal and frontal cortices [Frisoni et al., 2010]. Aging is the primary risk-factor in AD and leads to patterns of structural loss overlapping the pathological ones. However, the magnitude of brain atrophy due to $\mathrm{AD}$ is generally striking when compared to normal aging. As claimed in previous studies, AD is more likely to be a pathological state concurrent to aging, identified by specific biochemical and structural hallmarks [Nelson et al., 2011, Barnes, 2011].

Being able to separately model healthy aging and $\mathrm{AD}$ would allow us to to describe a given anatomy as being composed of distinct and concurrent factors. 
Such a decomposition would be extremely interesting not only to improve the understanding of the disease, but also for clinical purposes, such as for early diagnosis and for the development of drugs targetting the atrophy specific to the pathology. It is important to notice that, although brought on completely different biological mechanisms, aging and $\mathrm{AD}$ often map to common areas and correct identification of the respective contributions can be difficult, especially in morphometric studies. Moreover, it is plausible that these phenomena are not completely independent, and may overlap to create a positive "feedback" process. Thus, the onset of pathological changes may lead to accelerated global aging in the long term [Fjell et al., 2012], and vice versa.

A reliable estimate of the aging component is also important for modeling the evolution of the disease and for subsequent statistical analysis. When comparing the longitudinal observations from different clinical groups, at different aging stages, it is crucial to correctly position the observations on the time axis. This is not straightforward since the disease appears at different ages and chronologically older brains may have greater structural integrity than younger ones affected by the pathology. Therefore, it might be of practical interest to compute an index of age shift "relative" to a reference anatomical model.

The idea of modeling the time course of $\mathrm{AD}$ with respect to clinical and demographic factors was proposed in previous statistical studies [Yang et al., 2011, Ito et al., 2012, Samtani et al., 2012]. However, these works were limited to scalar observations such as clinical scores and demographics and thus do not provide an explicit model which relates structural changes in the entire brain to the disease and aging. Moreover, the disease progression was identified by clinical measures and was not therefore explicitly associated with a temporal time course. 
Although imaging-based surrogate measures of aging have been provided by different methodological studies [Franke et al., 2010, Davatzikos et al., 2009, Konukoglu et al., 2013], the idea of separately investigating aging and residual morphological changes has not been proposed before.

The objective of this work is to introduce a framework to identify and disentangle the brain anatomical changes related to normal aging from those related to other biological processes, such as AD. In particular, our framework is based on the hypothesis which relates the development of $\mathrm{AD}$ to the abnormal accumulation of beta-amyloid (A $\beta$ ) peptide in the brain [Jack et al., 2010]. We thus define "normal aging" as the morphological brain evolution which is not due to $\mathrm{A} \beta$. This evolution is modeled by non-linear registration and is used as a reference to characterize observed anatomy as a contribution from normal morphological aging (normal aging process) plus a specific morphological process which encodes the subject's specific variability such as pathological traits. We test our framework on healthy participants positive to the CSF A $\beta 42$ marker, in participants affected by mild cognitive impairment (MCI) and in AD patients.

The method is based on diffeomorphic non-linear registration and is detailed in Section 2. In Section 3 we show that such a framework provides a meaningful and accurate description of anatomical brain changes across the stages of $\mathrm{AD}$, characterized by increased morphological aging plus specific and local atrophy features.

\section{Methods}

The proposed method relies on specific modeling assumptions which are summarized here:

- The model of normal aging is derived from imaging data by applying a registration-based protocol detailed in Section 2.1. In particular, we as- 
sume that normal aging can be modeled through non-linear registration as a smooth and continuous process that can be extrapolated backward and forward in time beyond the observed imaging follow-up time. Moreover, we assume that normal aging is a constant process in time, i.e. that it does not accelerate or decelerate with respect to the biological age of the elderly population. We show in Sections 3.4 and 3.5 that these simple assumptions lead to plausible experimental results when compared with imaging and clinical data, and that the proposed model is a generalization of the classical linear mixed-effect (LME) modeling of univariate data used in longitudinal studies [Fitzmaurice et al., 2011].

- We define the specific morphological process as the remainder of the observed anatomy modulo the normal aging process. Thus, the specific morphological process encodes the morphological traits that cannot be described by the model of normal aging. In this study we aim to show that this specific process provides valuable information for discriminating pathological traits specific to $\mathrm{AD}$ across the whole disease time span (Section 3.6).

The framework was developed in the following way. We want to model the anatomy represented by a magnetic resonance image, $I_{k}$, acquired for a given subject $k$. For this purpose we describe the anatomical changes with respect to a reference anatomical template, $T$, through non-linear image registration. This work is based on diffeomorphic registration parameterized by stationary velocity fields [Lorenzi et al., 2013]. The non-linear registration setting estimates one-to-one smooth deformations that spatially align the anatomies represented by pairs of images. These deformations are completely identified voxel-wise by tangent velocities in the deformation space [Arsigny et al., 2006]. 
As illustrated in Figure 1, we parameterize the subject-to-template deformation $\phi_{k}$ by the flow of a stationary velocity field (SVF) $w_{k}$, which is denoted $\phi_{k}=\exp \left(w_{k}\right)$. In this framework the observed anatomical changes are entirely encoded in the SVF $w_{k}$.

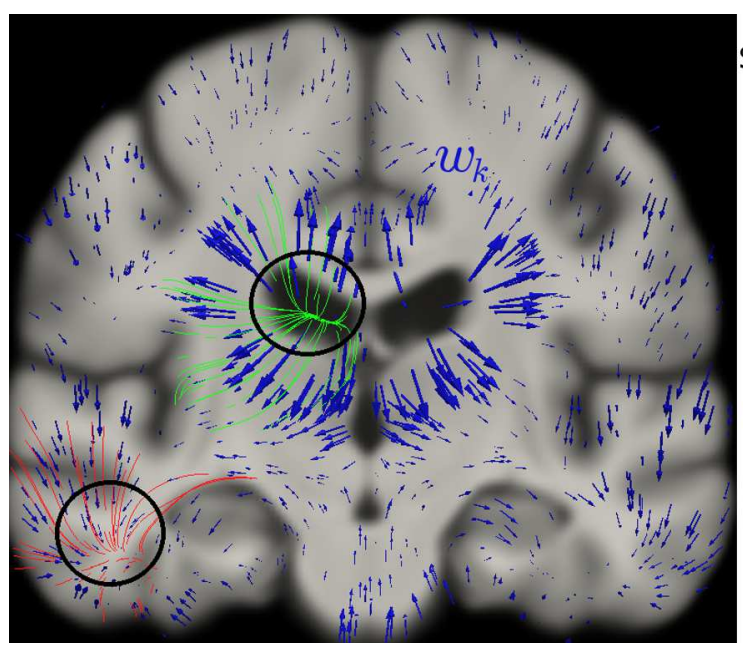

Stationary velocity field parameterization of deformations

Figure 1: The SVF $w_{k}$ (blue arrows) parameterizes the underlying transformation. In particular, the final displacement is obtained by locally following the flow-lines (as shown for instance by the green and red streams) identified by the SVF. The flux of the SVF across the boundaries of regions (e.g. black circles in the figure) measures the apparent regional volume change encoded by the deformation.

Since the space of SVFs is a linear vector space (contrarily to the space of deformations that it generates), we assume that $w_{k}$ can be decomposed into the algebraic sum of the normal deformation parameter $w_{a g e}^{k}$ plus a specific deformation parameter $w_{\text {specific }}^{k}$ (Figure 2). 


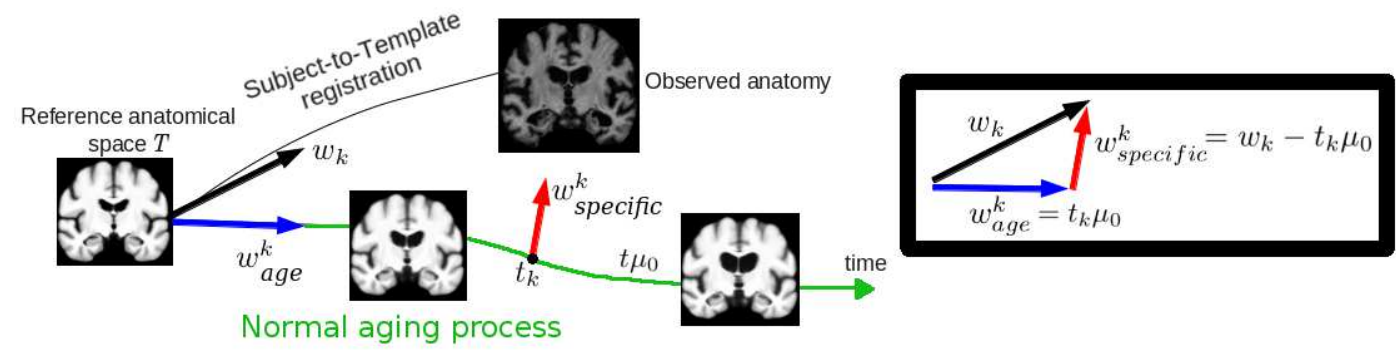

Figure 2: An observed anatomy can be described in terms of normal aging plus a specific morphological process.

The proposed framework analyzes these different components by processing the observed anatomy in different modeling steps as described in the following sections.

\subsection{Estimation of the Normal Aging Model}

This section describes the procedure based on non-linear registration used to create the reference model of the average group-wise longitudinal changes in healthy controls. As in the case of classical univariate linear mixed-effects modeling, we define a hierarchical model in which the subject specific trajectories of morphological changes are random realizations of an underlying normal aging process $\mu_{0}$ (Figure 3 ). The model is defined by the following levels:

1. Given the SVF $\mu_{0}(t)$ describing the group-wise aging process in the template space $T_{0}$, the subject-specific trajectories of healthy controls (in the template space) are realizations $\mu_{0}(t)+\epsilon^{S}$, where $\epsilon^{S}$ is the random variation from the fixed effect $\mu_{0}$.

2. The SVF describing the subject-specific trajectory $\mu^{S}(t)$ is obtained by 
an inter-subject spatial change of coordinates from the template space to the subject-specific coordinate system.

3. The trajectories $\mu^{S}(t)$ at points $t_{i}$ describe a time series of longitudinal images $I_{t_{i}}^{S}$. In particular we assume that the acquisition baseline time of each subject is the same within the population $\left(t_{0}=0\right)$, which equates to assuming that the aging progression is constant with respect to biological age.

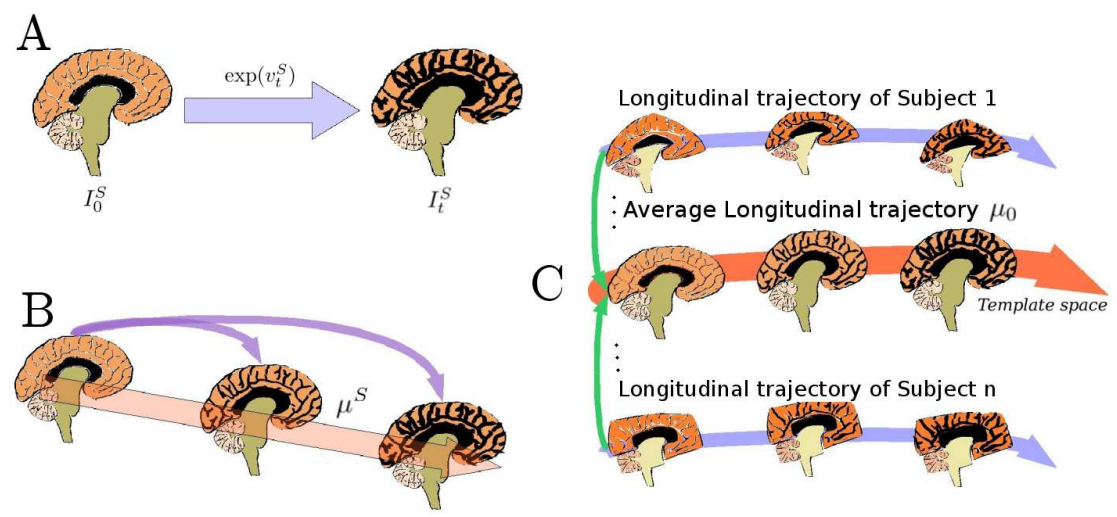

Figure 3: Hierarchical framework for the estimation of the group-wise model of normal aging. For a given subject, the follow-up longitudinal images are non-linearly registered to the baseline to estimate the associated SVF (A) . The subject-specific trajectory $\mu^{S}$ of the longitudinal morphological progression is estimated from the series of SVF with a linear model in time (B). Finally the group-wise model is computed as the mean of the transported subject-specific trajectories $(\mathrm{C})$.

Given the observed subject-specific trajectories of the anatomical changes defined by the series $I_{t_{i}}^{S}$, we estimate the group-wise trajectory $\mu_{0}$ by solving the inverse problem specified by the above hierarchical model (Figure 3). Please 
note that, as for linear mixed-effects modeling, our model assumes that the errors of the subject-to-template registration are similar for all the subjects. The model allows as well missing data and unequal sampling of trajectories by opportunely modeling inter and intra-subject variability. Details of the practical estimation of the normal aging trajectory $\mu_{0}$ using non-linear registration are given in Appendix A.

In the following sections we assume that normal aging evolves linearly in time according to the average group-wise $\mathrm{SVF} \mu_{0}$, so that it is parameterized by the trajectory $\mu_{0}(t)=t \mu_{0}$. Note that the resulting longitudinal normal aging process $\exp \left(t \mu_{0}\right)$ is non-linear.

\subsection{Identification and Extraction of the Morphological Age Shift}

Now that we have defined the normal aging model in a longitudinal setting, let us switch to a purely cross-sectional design: given an image $I_{k}$ of a subject $k$, we want to differentiate between the morphological patterns due to normal aging and those related to different biological processes.

Given the trajectory $t \mu_{0}$, we want to project the subject's anatomy $I_{k}$ on the "closest" point of the trajectory $T \circ \exp \left(t \mu_{0}\right)$ in order to determine its relative progression stage $t_{k}$ with respect to the normal aging process (Figure 2). By projecting the SVF which parameterizes the subject-to-template deformation $\exp \left(w_{k}\right)$ orthogonally onto the normal aging trajectory ${ }^{1}$, we obtain the decomposition $w_{k}=w_{\text {age }}^{k}+w_{\text {specific }}^{k}=t_{k} \mu_{0}+w_{\text {specific }}^{k}$.

In such a decomposition the time point $t_{k}$ is the morphological age shift of the subject $k$ with respect to the template $\mathrm{T}$ (which has the average age of the healthy control population), defined by the projection on the longitudinal model

\footnotetext{
${ }^{1}$ In the present work the projection is based on the standard $L^{2}$ metric.
} 
$t \mu_{0}$. The SVF $w_{\text {specific }}^{k}$ includes the specific morphological changes which cannot be related to the normal aging process (Figure 2). The time shift $t_{k}$ defines the projection on the longitudinal normal evolution $t \mu_{0}$ and is defined as the whole brain average of the voxel-by-voxel projections: $t_{k}=<w_{k}, \mu_{0}>_{(x)} /\left\|\mu_{0}\right\|_{(x)}^{2}$. Once $t_{k}$ is determined, the specific deformation parameter is simply defined voxel-wise as $w_{\text {specific }}^{k}=w_{k}-t_{k} \mu_{0}$. The removal of the normal deformation parameter $t_{k} \mu_{0}$ allows us to directly compare the remaining parameter $w_{\text {specific }}^{k}$ across subjects.

\subsection{Identification and Analysis of the Specific Morpholog- ical Process}

Let us turn now to the analysis of the specific deformation parameter $w_{\text {specific }}^{k}$ across subjects. This component defines the morphological changes that cannot be attributed to the normal aging process.

\section{Divergence Associated with the Specific Deformation Parameter}

Our diffeomorphic registration encodes observed morphological changes as dense diffeomorphic deformation fields parameterized by SVF. Volume changes are measured in a closed region by the flux of SVF across the boundary: the inward (resp. outward) flow of vectors across the boundary measures the apparent volume loss (resp. gain) (see Figure 1 for an illustrative example). More precisely, these local atrophy/growth processes are quantified by a scalar map: the divergence $\nabla \cdot w_{\text {specific }}^{k}$ associated with the specific deformation parameter $w_{\text {specific }}$ [Lorenzi et al., 2012] . 


\section{Discriminative Analysis of the Specific Deformation Parameter}

We test the ability of the divergence maps $\nabla \cdot w_{\text {specific }}$ to discriminate between a set of patients $P$ and a control group $C$. Since this is a scalar map we can use classical univariate methods.

We calculated the voxel-by-voxel map of the effect size associated with the average group-wise divergence $E S=\left(\operatorname{mean}\left(\nabla \cdot w_{\text {spec }}^{P}\right)-\operatorname{mean}\left(\nabla \cdot w_{\text {spec }}^{C}\right)\right) / \operatorname{sd}(\nabla \cdot$ $\left.w_{\text {spec }}^{P}\right)$, which quantifies the difference between volume changes modeled in patients and controls. We then chose a set of relevant regions for AD (hippocampi, medial temporal lobes (MTL), posterior cingulate (PC), and ventricles), where we identified the voxels of maximal positive and negative effect sizes. Regions were propagated in our reference space from the AAL labelled MNI template [Tzourio-Mazoyer et al., 2001]. These voxels were inflated and symmetrized in order to define a set of group-wise most discriminative regions. Finally Fisher's discriminant analysis was performed on the corresponding regional average divergence values associated with patients and controls. The discriminative analysis was performed by leave-one-out cross validation (500 permutations).

\section{$3 \quad$ Experiments and Results}

\subsection{Experimental Data}

Data used in the preparation of this article were obtained from the Alzheimers Disease Neuroimaging Initiative (ADNI) database (adni.loni.usc.edu). The ADNI was launched in 2003 by the National Institute on Aging (NIA), the National Institute of Biomedical Imaging and Bioengineering (NIBIB), the Food and Drug Administration (FDA), private pharmaceutical companies and non-profit organizations, as a $\$ 60$ million, 5-year public-private partnership. The Principal Investigator of this initiative is Michael W. Weiner, MD, VA Medical Center 
and University of California San Francisco. ADNI is the result of efforts of many co-investigators from a broad range of academic institutions and private corporations, and participants have been recruited from over 50 sites across the U.S. and Canada. For up-to-date information, see www.adni-info.org.

\subsection{Baseline Characteristics}

We chose ADNI structural MRIs from 65 healthy participants with normal levels of CSF A $\beta 42(>192 \mathrm{pg} / \mathrm{ml}$, group $\mathrm{A} \beta$-); 40 healthy participants with abnormal levels (group $\mathrm{A} \beta+$ ); 86 participants with mild cognitive impairment who subsequently converted to $\mathrm{AD}$ (group $M C I_{\text {conv }}$ ); $110 \mathrm{MCI}$ participants who remained stable during the observation period (group $M C I_{\text {stable }}$ ); and $134 \mathrm{AD}$ patients (group $A D$ ). Demographic as well clinical information were based on ADNI data updated to March 2012, with a follow-up period of 3 years from baseline. Baseline sociodemographical and clinical information for the different groups are shown in Table 1.

\begin{tabular}{|ccccccc|}
\hline & Healthy $\mathrm{A} \beta$ - & Healthy $\mathrm{A} \beta+$ & MCI stable & MCI converters & AD & p-value \\
\hline \hline \# participants & 65 & 40 & 110 & 86 & 134 & \\
\hline Age at baseline & $74.94(5.31)$ & $76.29(5.29)$ & $75.05(7.42)$ & $73.79(7.45)$ & $75.14(7.38)$ & 0.49 \\
\hline Education & $15.46(2.76)$ & $15.91(3.28)$ & $15.62(2.82)$ & $15.87(2.79)$ & $14.77(3.08)$ & 0.02 \\
\hline Gender (\% males) & 54 & 54 & 68 & 70 & 52 & 0.05 \\
\hline MMSE & $28.96(1.04)$ & $29.18(0.93)$ & $27.6(1.8)$ & $26.74(1.59)$ & $23.44(1.9)$ & $<0.001$ \\
\hline Modified ADAS-cog & $6.18(2.85)$ & $7.08(3.18)$ & $10.24(4.16)$ & $12.92(4.43)$ & $18.27(5.98)$ & $<0.001$ \\
\hline APOE4 (\% positives) & 10 & 48.6 & 49.4 & 73 & 66 & $<0.001$ \\
\hline
\end{tabular}

Table 1: Average baseline socio-demographical and clinical scores for the participants included in the study (standard deviations in parenthesis). Last column: p-values for the differences between averages across groups (Anova). 


\subsection{Analysis of Normal Aging and Specific Morphological Processes}

Previous studies show that healthy elderly subjects with pathological CSF A $\beta 42$ levels $(<192 \mathrm{pg} / \mathrm{ml})$ have a more pronounced brain atrophy progression [Fjell et al., 2010, Tosun et al., 2010], which might be a marker of the pre-symptomatic stage of $\mathrm{AD}$. We therefore defined the normal aging model by considering only the $\mathrm{A} \beta$ - group as a reference normal population. The longitudinal observations (baseline, 6, 12, 24 and 36 months) available for the $\mathrm{A} \beta$ - group were considered to model a reference healthy evolution $\mu_{0}$ normalized to an anatomical reference template $T$ according to Section 2.1. The average follow-up time for the $\mathrm{A} \beta$ group was 18.32 months $( \pm 11.47)$ and the average number of follow-up acquisitions per participant was $4.46( \pm 0.8)$ : 40 participants had 5 scans (thus the complete set of longitudinal images), 17 had 4 scans, 6 had 3 scans, and only 2 had 2 scans.

The template $T$ was estimated as in [Guimond et al., 2000] from the pooled healthy group. Therefore, the reference age of the template is 75.41 years, which corresponds to the average age of healthy participants.

In order to unbias the analysis with respect to the healthy $\mathrm{A} \beta$ - population, we centered the SVFs by subtracting the average subject-to-template SVF of the A $\beta$ - group. The unbiased SVF were then analyzed by following the framework proposed in Sections 2.2 and 2.3. The effectiveness of the specific morphological process in encoding information relevant to the pathology was tested by performing two different discriminative analyses for the classification between $\mathrm{AD}$ vs. healthy, and $M C I_{\text {conv }}$ vs. $M C I_{\text {stable }}$. 


\subsection{Longitudinal Model of Normal Aging}

The reference model of normal aging estimated for the $\mathrm{A} \beta$ - group is shown in Figure 4A. The estimated normal aging process is characterized mainly by ventricular growth and by a corresponding apparent atrophy in surrounding regions. Volume loss is widespread in temporal areas, and characterizes the shrinkage of hippocampi. Interestingly, the model displays an important atrophy pattern in the cerebellum, which is in agreement with observations previously reported in the literature [Koller et al., 1981, Torvik et al., 1986, Luft et al., 1999]. Finally, other regions of volume loss were localized in the superior frontal cortex and in the cuneus, in agreement with previous findings on longitudinal atrophy in the ADNI healthy cohort [Fjell et al., 2009]. 

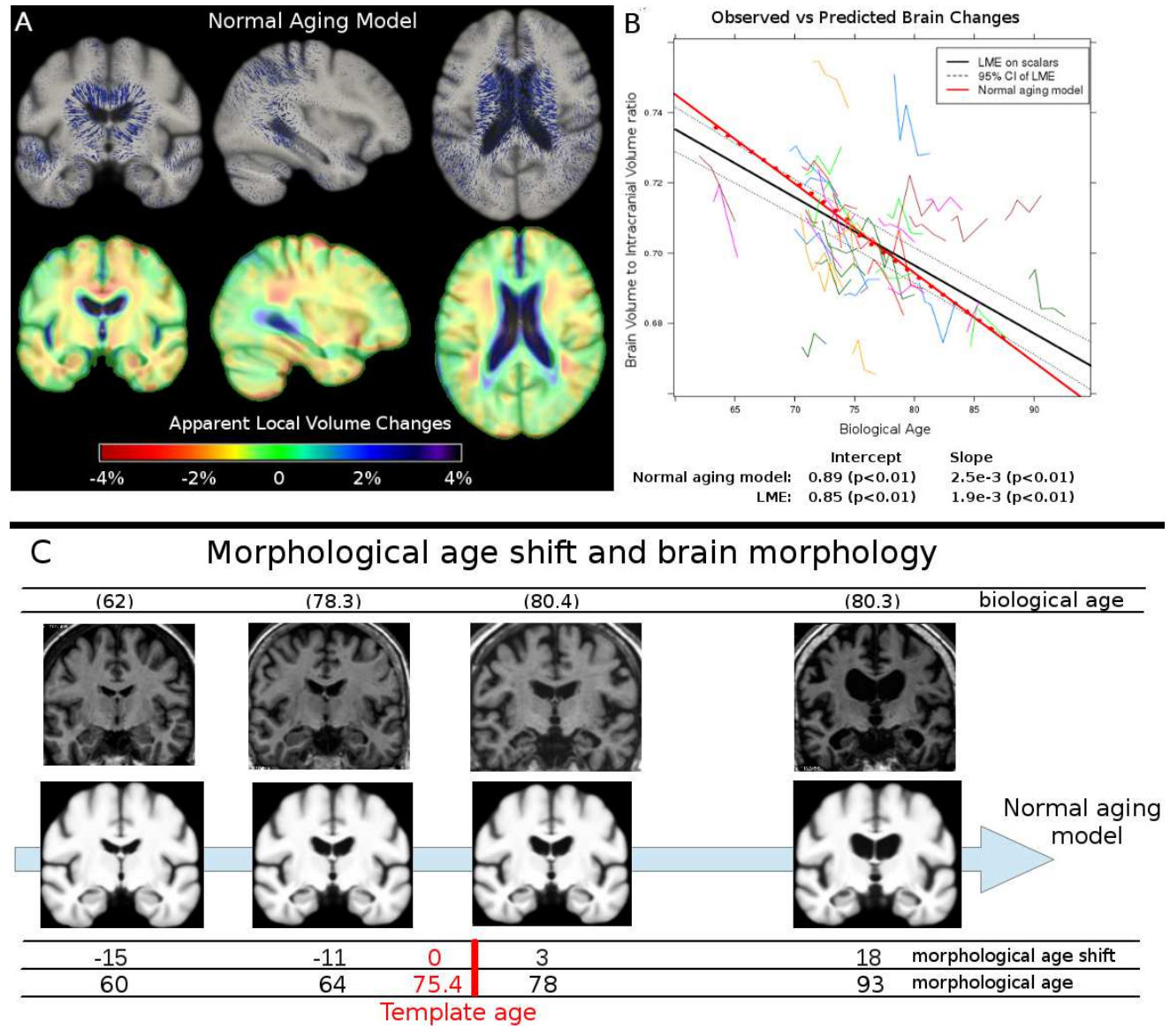

Figure 4: A) Normal aging model. Top: The average SVF describes the groupwise trajectory of normal aging observed in the healthy $A \beta$ - group (blue arrows indicate magnitude and direction of the trajectory). Bottom: Average 1-year volume changes associated to the model of normal aging. The model leads to local relative volume changes ranging from around $+4 \%$ for the expansion of ventricles, to $-4 \%$ for the relative volume loss in temporal areas. B) Observed vs modeled brain volume changes in the $\mathrm{A} \beta$ - cohort. Black line: fitted slope (and 95\% confidence interval) for the linear mixed-effects model (LME) on the observed subject-specific trajectories of scalar volumes (colored lines). 
Figure 4: (Previous page.) Red dots: Progression of brain volume changes associated to the normal aging model and associated fitted slope (red line). Brain volume is quantified here by the ratio between grey+white matter tissue and total intracranial volume (grey+white+CSF areas). C) Modeled longitudinal evolution extrapolated from -15 to 18 years, and corresponding observed normal anatomies with estimated morphological age and age shift (biological age in parenthesis).

From the quantitative point of view the average relative annual volume change in selected ROIs was $-0.54 \%$ and $-0.4 \%$ for hippocampi and medial temporal lobes respectively, and $+1.3 \%$ in the ventricles. These results are compatible with those reported in several longitudinal morphometric studies [Fjell et al., 2009, Risacher et al., 2010, Fox and Schott, 2004, Jack et al., 2004].

Figure $4 \mathrm{~B}$ provides a statistical assessment of the ability of the proposed aging model to describe the observed group-wise longitudinal anatomical changes. We compared the change in brain volume associated with the normal aging model, Figure 4A, to the longitudinal brain volume changes measured in the $\mathrm{A} \beta$ - group. Brain volume was quantified as the ratio between grey+white matter tissue and total intracranial volume (grey+white+CSF areas). Tissue segmentation was performed with the FAST tool available in the FSL suite [Zhang et al., 2001].

For the sake of comparison we also compared the progression associated with our model to the classical linear mixed-effect modeling (LME) of the scalar measures of brain volumes. We observed that the brain volume progression associated with the model of normal aging is close to that modelled by LME, and almost entirely falls into the $95 \%$ confidence interval within the age range $[65,90]$. These results indicate that the hierarchical model described in Section 2 is compatible with classical approaches of longitudinal analysis, while providing a more comprehensive description of the whole brain dynamics associated with morphological change. 
Figure $4 \mathrm{C}$ shows the extrapolation of the longitudinal model in time. The progression follows the model highlighted in Figure 4A and is in fact characterized by major ventricles enlargement and by mild atrophy spread in the temporal areas.

\subsection{Morphological Age Shift}

Figure $4 \mathrm{C}$ provides a qualitative illustration of morphological age estimated with respect to the normal aging model. We see that the morphological age shift correctly temporally aligns the subject's brains relative to the aging model.

The statistical analysis reported in Table 2 shows that the morphological age shift is significantly correlated with the biological one, and with neuropsychological scores. Men have a significantly higher morphological age shift than women. This result is consistent with previous studies reporting a general accelerated aging process in men across the whole life span [Coffey et al., 1998, Mann et al., 2011].

\begin{tabular}{|ccc|}
\hline & Pearson's Correlation & p-value \\
\hline \hline $\begin{array}{c}\text { Age } \\
\text { at baseline }\end{array}$ & $\mathbf{0 . 4}$ & $<0.001$ \\
\hline Education & $\mathbf{0 . 0 9}$ & 0.05 \\
\hline $\begin{array}{c}\text { Gender } \\
\text { (Male=1, Female=0) }\end{array}$ & $\mathbf{0 . 2 5}$ & $<0.001$ \\
\hline MMSE & $\mathbf{- 0 . 2}$ & $<0.001$ \\
\hline $\begin{array}{c}\text { ADAS-cog } \\
\text { (modified) }\end{array}$ & $\mathbf{0 . 2 3}$ & $<0.001$ \\
\hline $\begin{array}{c}\text { APOE4 } \\
\text { (\# alleles) }\end{array}$ & -0.04 & 0.4 \\
\hline
\end{tabular}

Table 2: Pearson's correlation between socio-demographical/clinical scores and morphological age shift (in bold significant correlation).

Finally, positive Apoe4 is not generally correlated with the morphological age shift, even though a significant negative correlation was measured in healthy participants $\mathrm{A} \beta$-, and in stable MCI (Supplementary Table S2).

Interestingly, Figure $4 \mathrm{C}$ shows that the morphological age shift is only par- 
tially related to biological aging. In fact, more pronounced anatomical changes are depicted here in advanced stages of the model, and thus they identify virtually older anatomies.

For this reason, even though the considered groups did not significantly differ in age, the morphological age shift increases as the clinical condition gets closer to $\mathrm{AD}$. As shown in Figure $5, \mathrm{~A} \beta+, \mathrm{MCI}$ and $\mathrm{AD}$ become virtually older when compared to healthy $\mathrm{A} \beta$ - (p-values in the boxes). $M C I_{\text {conv }}$ are significantly older than $M C I_{\text {stable }}(\mathrm{p}=0.035)$, indicating a possible accelerated aging process induced by the progression of $\mathrm{AD}$.

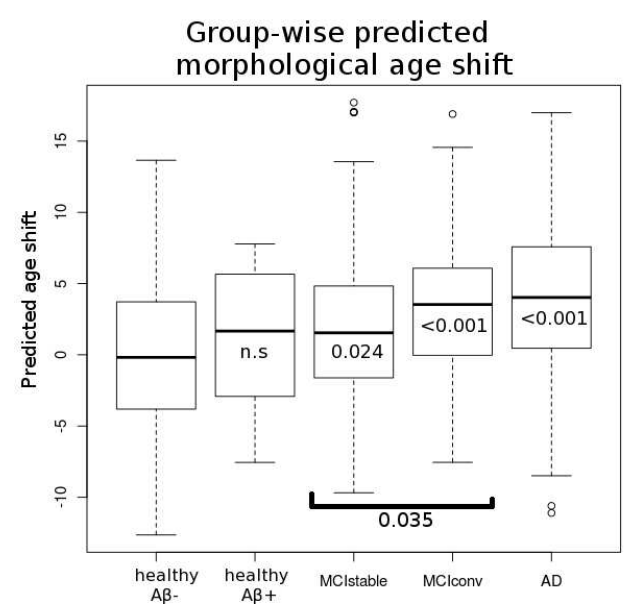

Figure 5: Box-plot of the group-wise morphological age shift estimated for the clinical groups. The morphological age shift describes statistically significant older brains (standard t-test, p-value in the boxes) with respect to the healthy $\mathrm{A} \beta$ - for all the patient groups. Interestingly, MCI converters are "virtually older" than the MCI stables $(\mathrm{p}=0.035)$. 


\subsection{Analysis of the Specific Individual Process}

Figure 6 shows the average specific deformation parameters $w_{\text {specific }}^{k}$ associated with different groups. The morphological changes specific to healthy $\mathrm{A} \beta+$ are mild, while the changes specific to the MCI converters are more pronounced and map to the frontal cortex, ventricles, temporal poles, entorhinal cortex and hippocampi. The same pattern is observed in AD patients. 

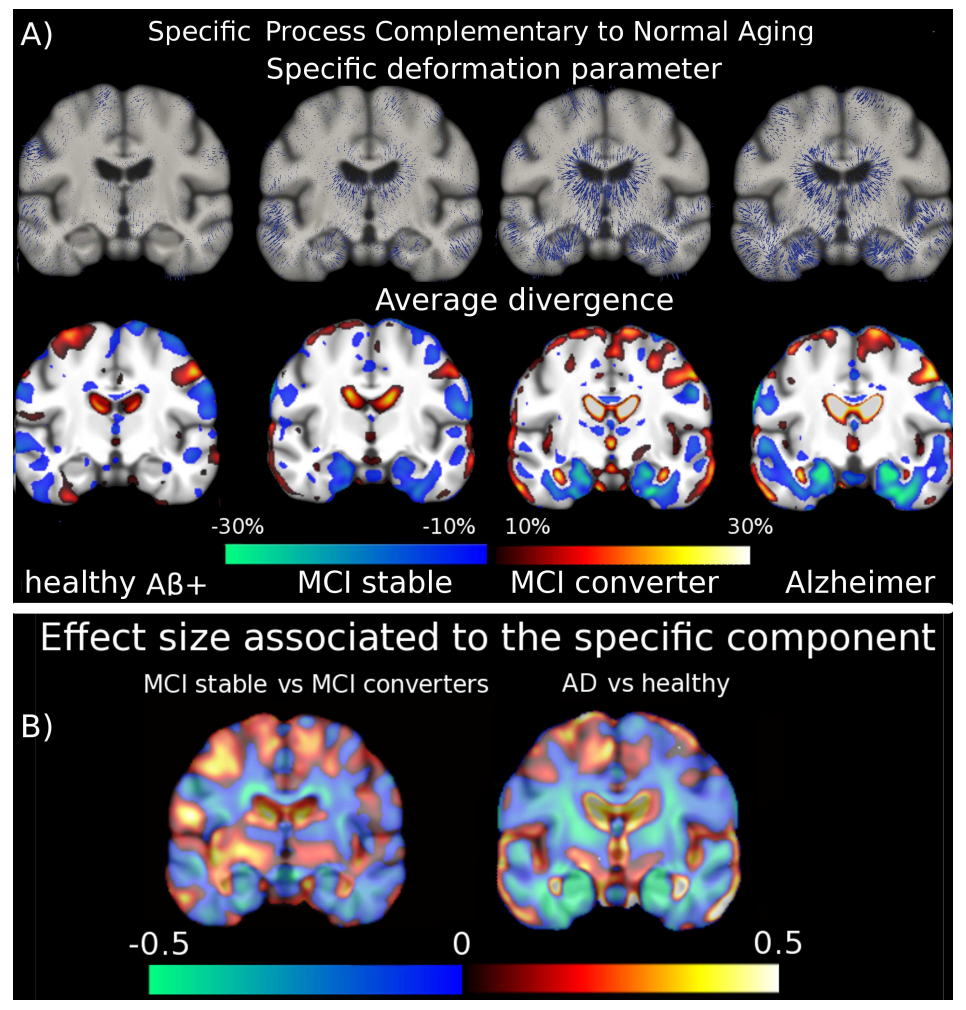

Figure 6: A) First row: Average specific deformation parameter not related to healthy aging (blue arrows indicate magnitude and direction of the deformation). MCI converters and AD patients show a more pronounced pattern of morphological change mapping mainly to ventricles, temporal poles, the entorhinal cortex and hippocampi. Second row: associated relative volume change. Warm colors: apparent growth. Cool colors: apparent atrophy. B) Effect size associated with the divergence maps of the specific deformation parameter. The effect size quantifies the magnitude of the difference between volume changes modeled in the two groups. AD patients and healthy controls show greater differences localized in hippocampi, temporal areas, and in the ventricles. These differences are milder when comparing MCI converters with stable. 
In Figure 6A, second row, we notice that changes in clinical condition (from $\mathrm{A} \beta+$, to $M C I_{\text {conv }}$ and $\mathrm{AD}$ ) are associated with larger and more intense atrophy patterns. We notice an increasing pattern of growth in the CSF regions, as well as a complementary pattern of atrophy mapping onto hippocampi, temporal areas and cortices. Interestingly, the average atrophy patterns of the specific parameters are very similar and compatible with those associated with the full SVF (Supplementary Figure S7).

Figure $6 \mathrm{~B}$ shows the effect size between the divergence maps of MCI converters vs stable, and of $\mathrm{AD}$ vs healthy controls. As expected, the effect size between $\mathrm{AD}$ and healthy controls is higher than that between MCI converters and stables, indicating the larger variability in the MCI group. In Supplementary Figure S7 we show that the effect size obtained from the specific atrophy component is slightly higher (around ventricles and in the white matter) than the one obtained with the whole SVF (thus when considering normal aging + specific deformation processes) when comparing MCI stable vs converters. When comparing $\mathrm{AD}$ with healthy controls, the effect size associated with the whole SVF is generally higher in white matter, and mildly lower in the temporal horns of the hippocampi.

Finally, Table 3 shows regional and pooled accuracy of the specific process in differentiating $A D$ vs $C t r l s$, and $M C I_{\text {conv }}$ vs $M C I_{\text {stable }}$. The fair classification results ( $91 \%$ sensitivity, $84 \%$ specificity for $\mathrm{AD}$ vs $\mathrm{Ctrls}$, and $67 \%, 63 \%$ for $M C I_{\text {conv }}$ vs $\left.M C I_{\text {stable }}\right)$ indicate the ability of the specific parameter to encode information relevant to the disease condition. The provided classification results are significantly better than those given by pure chance $(p<0.001$, McNemar's Chi-Square test), and are in line with those available in the literature on the ADNI dataset [Cuingnet et al., 2011, Chincarini et al., 2011]. We have thus shown that the specific deformation parameter encodes specific pathological 
traits across the whole disease time span, which are significantly discriminative among the considered populations.

\begin{tabular}{|l|cccc|cccc|}
\hline & \multicolumn{4}{|c|}{ AD vs Ctrls } & \multicolumn{4}{c|}{$M C I_{\text {conv }}$ vs $M C I_{\text {Stable }}$} \\
\hline & Sens & Spec & PPV & NPV & Sens & Spec & PPV & NPV \\
\hline \hline All features & $\mathbf{9 1}$ & $\mathbf{8 4}$ & $\mathbf{8 5}$ & $\mathbf{9 0}$ & 54 & 54 & 54 & 54 \\
\hline MTL (-) & 86 & 81 & 85 & 82 & 53 & 51 & 52 & 52 \\
\hline MTL (+) & 73 & 77 & 76 & 74 & 57 & 57 & 57 & 57 \\
\hline Hippocampi (-) & 77 & 71 & 75 & 73 & 55 & 47 & 51 & 51 \\
\hline Hippocampi (+) & 77 & 63 & 73 & 67 & $\mathbf{6 7}$ & $\mathbf{6 3}$ & $\mathbf{6 4}$ & $\mathbf{6 5}$ \\
\hline Ventricles (+) & 65 & 69 & 68 & 66 & 61 & 43 & 52 & 52 \\
\hline Ventricles (-) & 68 & 69 & 69 & 68 & 58 & 56 & 57 & 57 \\
\hline PC (-) & 58 & 59 & 59 & 59 & 58 & 58 & 58 & 58 \\
\hline PC (+) & 59 & 50 & 54 & 54 & 47 & 74 & 64 & 58 \\
\hline
\end{tabular}

Table 3: Regional classification accuracy for the leave-one-out discrimination. The analyzed features are the positive and negative flux ( + and - ) of the specific parameter across the regions of interest.

\section{Conclusions}

We proposed a method to describe brain anatomy as contributions of two independent processes: morphological aging and a specific component. These components identify different clinical stages, and are compatible with the hypothesis that points to the abnormal levels of CSF A $\beta 42$ as a presymptomatic marker of $\mathrm{AD}$ in the early stages.

We showed that more advanced AD stages (from $\mathrm{A} \beta+$ to $\mathrm{MCI}$ converters, and finally to AD) are associated with both "virtually older" brains, and with increased specific morphological changes not related to the normal aging process. Thus, according to our model, AD might have an influence on the overall aging of the brain acting as an acceleration factor. 


\section{Relationship with classical morphometric studies}

When compared to standard analysis approaches of group-wise structural changes such as classical voxel/tensor-based morphometry [Hua et al., 2008, Good et al., 2002], or discriminative analysis [Cuingnet et al., 2011, Chincarini et al., 2011], our method has the advantage that provides complementary information representing morphological aging and a specific deformation parameters which carries relevant biological meaning. For this reason, the proposed method provides a novel way of interpreting morphometric results. For instance, it may be of great interest to investigate the relationship between the morphological age shift and specific changes in characterizing brain structural and clinical reserve in preclinical/prodromal stages.

\section{Combining cross-sectional and longitudinal analysis}

Our method proposes cross-sectional comparison of brain images by means of a previously defined longitudinal model of morphological changes.

The statistical modeling of the inter-subject variability in computational anatomy is challenging, and the interpretation of group-wise comparison results is always bound to the statistical assumptions about the measured features (for instance concerning the statistical distribution of the Jacobian/divergence values). In this study we showed that by removing the aging component we increase the ability in detecting specific group-wise differences. For this reason the proposed method represents a novel and promising approach to the appropriate modeling and interpretation of group-wise anatomical variation.

We note in fact that the voxel-by-voxel analyis of the divergence maps in Section 3.6 is compatible with standard voxel/tensor-based morphometry approaches, and leads to very similar results to those provided by classical groupwise comparison [Hua et al., 2008, Good et al., 2002]. It is, however, providing 
a slightly higher effect size when comparing MCIs. This indicates that the removal of the normal aging process could enhance the estimation of pathological volume changes. When comparing $\mathrm{AD}$ to healthy controls the analysis of the specific morphological process provides a slightly larger effect size in the temporal horns, but generally lower in the white matter. This latter result can be explained by noting that the removal of the aging process aims to decorrelate the morphological changes explained by the aging model. In this way we reduce, for instance, the effect of ventricles expansion, detectable in TBM as an apparent contraction in the white matter.

\section{Interpretation of aging and specific processes}

Concerning the modeling of the specific deformation parameter we note that by definition this component is highly heterogeneous across the population since it includes normal anatomical variability as well as pathological features. In this study we have shown that in spite of this high variability, the specific deformation is able to accurately describe anatomical features specific to AD. The discriminative analysis performed in Section 3.6 showed that the specific deformation parameter includes specific pathological traits which characterize the whole disease time span. Future studies based on more sophisticated machine learning techniques, as proposed for instance in [Cuingnet et al., 2011, Chincarini et al., 2011], may lead to improved classification results.

In our model the morphological age shift is based on the whole brain average of the projection on the normal aging model. Here we make a precise assumption about the aging process, which is in fact defined globally. Therefore, accelerated aging is constrained with respect to the whole brain normal aging model, and any local departure from it (for instance in some specific regions), is interpreted as a specific morphological change, independent from aging. Different MRIbased indices of brain aging have been proposed in the past [Franke et al., 2010, 
Davatzikos et al., 2009]. Our model integrates these approaches into a more general description of the $\mathrm{AD}$ process. We note that the morphological age shift for $\mathrm{AD}$ patients is lower than the aging score estimated in [Franke et al., 2010] (4 vs 10 years). In fact we have shown that $\mathrm{AD}$ is characterized by a more pronounced specific and concurrent pattern of atrophy. In this case, the present result motivates and provides clinical outcomes for the design of disease specific modifying drugs which do not have an impact on normal aging.

We observed a weak but significant positive correlation between morphological age shift and years of education. The correlation is significant and stronger when considered in healthy participants only (Supplementary Table S1). This latter finding could indicate that morphologically older participants with high education are more likely to appear cognitively healthy. Therefore the morphological age shift might represent a measure of cognitive and structural reserve in normal aging [Coffey et al., 1999].

The proposed average model of aging assumes that the baseline acquisition time is unique for the healthy cohort, as already proposed in previous longitudinal studies of brain volume changes in $\mathrm{AD}$, based on mixed-effects modeling [Ridha et al., 2006]. We showed in the experimental section that under these assumptions we obtained similar results to those provided by classical univariate LME models, by correctly describing the temporal variability of brain changes in the healthy cohort. However, it will be of interest to explicitly account for the inter-subject baseline differences in future studies. This amounts to modifying the assumption about the constant evolution in time of the healthy aging process.

Finally, the proposed model could be extended in future work in order to account for different evolution trajectories, and to explicitly model different neurodegenerative pathologies, and AD subtypes. 
To conclude, our approach provides new insights which may help the understanding of $\mathrm{AD}$ dynamics, and could thus represent a more precise instrument to identify outcomes in clinical trials for disease modifying drugs.

\section{Limitations}

Some methodological limitations should be considered in this study. The proposed model of aging progression is based on non-linear registration, and is therefore estimated from image data only. This means that no biological/biomechanical information was accounted in the definition of the average trajectory. Nonlinear registration is driven by image intensities, thus it only models apparent observable anatomical changes. Moreover, non-linear registration results are dependent on the registration model and may potentially provide different results depending on the choice of parameters, similarity measure and regularization scheme. However, we have already shown in previous work that LCC-Demons non-linear registration provides reliable and meaningful results when applied

to brain image registration problems, especially for the longitudinal analysis of atrophy [Lorenzi et al., 2013].

Finally, only three year of follow-up imaging data is available for the healthy cohort, and therefore the group-wise evolution was limited to a linear model in time for the SVF due to the lack of sufficient longitudinal observations.

\section{Acknowledgment}

The authors would like to thank Sheila and John Stark, and Kristin McLeod for they valuable help in manuscript proofreading.

This work was partially funded by the European Research Council (ERC advanced Grant MedYMA), ANR blanc Karametria and the EU project Care4Me.

Data collection and sharing for this project was funded by the Alzheimer's 
Disease Neuroimaging Initiative (ADNI) (National Institutes of Health Grant U01 AG024904). ADNI is funded by the National Institute on Aging, the National Institute of Biomedical Imaging and Bioengineering, and through generous contributions from the following: Alzheimers Association; Alzheimers Drug Discovery Foundation; BioClinica, Inc.; Biogen Idec Inc.; Bristol-Myers Squibb Company; Eisai Inc.; Elan Pharmaceuticals, Inc.; Eli Lilly and Company; F. Hoffmann-La Roche Ltd and its affiliated company Genentech, Inc.; GE Healthcare; Innogenetics, N.V.; IXICO Ltd.; Janssen Alzheimer Immunotherapy Research \& Development, LLC.; Johnson \& Johnson Pharmaceutical Research \& Development LLC.; Medpace, Inc.; Merck \& Co., Inc.; Meso Scale Diagnostics, LLC.; NeuroRx Research; Novartis Pharmaceuticals Corporation; Pfizer Inc.; Piramal Imaging; Servier; Synarc Inc.; and Takeda Pharmaceutical Company. The Canadian Institutes of Health Research is providing funds to support ADNI clinical sites in Canada. Private sector contributions are facilitated by the Foundation for the National Institutes of Health (www.fnih.org). The grantee organization is the Northern California Institute for Research and Education, and the study is coordinated by the Alzheimer's Disease Cooperative Study at the University of California, San Diego. ADNI data are disseminated by the Laboratory for Neuro Imaging at the University of Southern California.

\section{Disclosure Statement}

The authors have nothing to disclose.

\section{References}

V. Arsigny, O. Commowick, X. Pennec, and N. Ayache. A log-Euclidean framework for statistics on diffeomorphisms. In Proc. of Medical Imaging Computing and Computer Assisted Intervention (MICCAI), pages 924-931, 2006. 
C. Barnes. Secrets of aging: What does a normally aging brain look like? Biol Rep., 3(22), 2011. Published online.

S. N. Burke and C. A. Barnes. Neural plasticity in the ageing brain. Nature Reviews Neuroscience, (7):30-40, 2006.

A. Chincarini, P. Bosco, P. Calvini, et al. Local MRI analysis approach in the diagnosis of early and prodromal Alzheimer's disease. NeuroImage, 58(2): $469-480,2011$.

C.E. Coffey, J.F. Lucke, J.A. Saxton, G. Ratcliff, L.J. Unitas, B. Billig, and R.N. Bryan;. Sex differences in brain aging: a quantitative magnetic resonance imaging study. Arch Neurol, 2(55):169-179, 1998.

C.E. Coffey, J.A. Saxton, G. Ratcliff, R.N. Bryan, and J.F. Lucke;. Relation of education to brain size in normal aging: implications for the reserve hypothesis. Neurology, 1(53):189-196, 1999.

R. Cuingnet, E. Gerardin, J. Tessieras, G. Auzias, S. Lehricy, M Habert, M. Chupin, H. Benali, and O. Colliot. Automatic classification of patients with Alzheimers disease from structural MRI: a comparison of ten methods using the ADNI database. NeuroImage, 56(2):766-781, 2011.

C. Davatzikos, F. Xu, Y. An, Y. Fan, and S.M. Resnik. Longitudinal progression of Alzheimer's-like patterns of atrophy in normal older adults: the SPAREAD index. Brain, 132(8):2026-2035, 2009.

G.M. Fitzmaurice, N.M. Laird, and J.H. Ware. Applied Longitudinal Analysis. Wiley, 2011.

A.M. Fjell, K.B. Walhovd, C.F. Notestine, L.K. McEvoy, D.J. Hagler, D. Holland, J.B. Brewer, and A.M. Dale. One-Year Brain Atrophy Evident in Healthy Aging. The Journal of Neurosciences, 29(48):15223-15231, 2009. 
A.M. Fjell, K.B. Walhovd, C.F. Notestine, and et al. Brain atrophy in healthy aging is related to csf levels of A $\beta 1-42$. Cereb. Cortex, 20-9, 2010.

A.M. Fjell, L.T. Westlye, H. Grydeland, I. Amlien, T. Espeseth, I. Reinvang, N. Raz, A.M. Dale, and K.B. Walhovd. Accelerating Cortical Thinning: Unique to Dementia or Universal in Aging? Cereb Cortex, pages 1-2, 2012.

N. Fox and J.M. Schott. Imaging cerebral atrophy: normal ageing to Alzheimer's disease. The Lancet, 363(9406):392-394, 2004.

K. Franke, G. Ziegler, S. Klöppel, and C. Gaser. Estimating the age of healthy subjects from T1-weighted MRI scans using kernel methods: Exploring the influence of various parameters. NeuroImage, 50(3):883-892, 2010.

G.B. Frisoni, N.C. Fox, C.R. Jack Jr, P. Scheltens, and P.M. Thompson. The clinical use of structural MRI in Alzheimer disease. Nat Rev Neurol, 6:67-77, 2010.

C. Good, R. Scahill, N.C. Fox, J. Ashburner, K.J. Friston, D. Chan, W.R. Crum, M.N. Rossor, and R.S.J. Frackowiak. Automatic differentiation of anatomical patterns in the human brain: Validation with studies of degenerative dementias. NeuroImage, 17(1):29-46, 2002.

A. Guimond, J. Meunier, and J.P. Thirion. Average brain models: A convergence study. Computer Vision and Image Understanding, 77-2, 2000.

P.R. Hof and C.V. Mobbs. Handbook of the neuroscience of aging. Elsevier, 1984.

Xue Hua, Alex D. Leow, Suh Lee, et al. 3D characterization of brain atrophy in Alzheimer's disease and mild cognitive impairment using tensor-based morphometry. NeuroImage, 41(1):19-34, 2008. 
K. Ito, M.M. Hutmacher, and B.W. Corrigan. Quantifying the pathophysiological timeline of Alzheimer's disease. Journal of Pharmacokinetics and Pharmacodynamics, 39(6):601-618, 2012.

C.R. Jack, M.M. Shiung, J.L. Gunter, P.C. O'Brien, S.D. Weigand, D.S. Knopman, B.F. Boeve, R.J. Ivnik, G.E. Smith, R.H. Cha, E.G. Tangalos, and R.C. Petersen. Comparison of different MRI brain atrophy rate measures with clinical disease progression in AD. Neurology, 62(4):591-600, 2004.

C.R. Jack, D.S. Knopman, W.J. Jagust, et al. Hypothetical model of dynamic biomarkers of the Alzheimer's pathological cascade . Lancet Neurol, 9(1): $119-128,2010$.

W.C. Koller, S.L. Glatt, J.H. Fox, A.W. Kaszniak, R.S. Wilson, and M.S. Huckman. Cerebellar atrophy: relationship to aging and cerebral atrophy. Neurology, 31(11):1846-8, 1981.

E. Konukoglu, B. Glocker, D. Zikic, and A. Criminisi. Neighbourhood approximation using randomized forests. Medical Image Analysis, 17(7):790-804, 2013.

X. Long, W. Liao, D. Liang, B. Qiu, and L. Zhang. Healthy aging: An automatic analysis of global and regional morphological alterations of human brain. Acad Radiol., 19(7):785-93, Jul 2012.

M. Lorenzi and X Pennec. Parallel transport with pole ladder: Application to deformations of time series of images. Journal of Mathematical Imaging and Vision, pages 1-2, 2013.

M. Lorenzi, N. Ayache, and X. Pennec. Regional flux analysis of longitudinal atrophy in Alzheimer's disease. In MICCAI, volume 7510 of LNCS, pages 739-746. Springer, 2012. 
M. Lorenzi, N. Ayache, G.B. Frisoni, and X. Pennec. LCC-Demons: a robust and accurate diffeomorphic registration algorithm. NeuroImage, 1(81):47083, Nov 2013.

A.R. Luft, M. Skalej, J.B. Schulk, D. Welte, R. Kolb, K. Burk, T. Klockgether, and K. Voigt. Patterns of Age-related Shrinkage in Cerebellum and Brainstem Observed In Vivo Using Three-dimensional MRI Volumetry. Cereb Cortex, 9 (7):712-721, 1999 .

S.L. Mann, E.A. Hazlett, W. Byne, P.R. Hof, M.S. Buchsbaum, B.H. Cohen, K.E. Goldstein, M.M. Haznedar, E.M. Mitsis abd L.J. Siever, and K.W. Chu;. Anterior and posterior cingulate cortex volume in healthy adults: effects of aging and gender differences. Brain Res, 1401(15):18-29, 2011.

P. T. Nelson, E. Head, F.A. Schmitt, P.R. Davis, J.H. Neltner, G.A. Jicha, E.L. Abner, C.D. Smith, L.J. Van Eldik, R.J. Kryscio, and S.W Scheff. Alzheimer's disease is not "brain aging": neuropathological, genetic, and epidemiological human studies. Acta Neuropathol., 121(5):571-587, 2011.

B.H Ridha, J. Barnes, J.W Bartlett, A. Godbolt, T. Pepple, M.N. Rossor, and N.C Fox. Tracking atrophy progression in familial Alzheimer's disease: a serial MRI study. Lancet Neurol, 5:828-834, 2006.

S.L. Risacher, L. Shen, J.D. West, S. Kim, B.C. McDonald, L.A. Beckett, D.J. Harvey, C.R. Jack, M.W. Weiner, and A.J. Saykin. Longitudinal MRI atrophy biomarkers: Relationship to conversion in the ADNI cohort. Neurobiol Aging, 31(9):1401-1418, 2010.

M.N. Samtani, M. Farnum, V. Lobanov, E. Yang, N. Raghavan, A. Dibernardo, and V. Narayan. An improved model for disease progression in patients from the Alzheimer's disease neuroimaging initiative. Journal of Clinical Pharmacology, 52(5):629-644, 2012. 
R.I. Scahill, J.M. Schott, J.M. Stevens, M.N. Rossor, and N.C. Fox. Mapping the evolution of regional atrophy in Alzheimer's disease: unbiased analysis of fluid-registered serial MRI. Proc Natl Acad Sci, 99:4703-4707, 2002.

E.R. Sowell, B.S. Peterson, P.M. Thompson, S.E. Welcome, A.L. Henkenius, and A.W. Toga. Mapping cortical change across the human life span. Nat Neurosci, 3(6):309-315, 2003.

P. Thompson, K.M. Ayashi, G. Zubicaray, A.L. Janke, S.E. Rose, J. Semple, D. Herman, M.S. Hong, S.S. Dittmer, D.M. Doddrell, and A.W. Toga. Dynamics of gray matter loss in Alzheimer's disease. The Journal of Neuroscience, 23:994-1005, 2003.

A. Torvik, S. Torp, and C.F. Lindboe. Atrophy of the cerebellar vermis in ageing: A morphometric and histologic study. Journal of the Neurological Sciences, 76(2):283-294, 1986.

D. Tosun, N. Schuff, D. Truran-Sacrey, and et al. Relations between brain tissue loss, CSF biomarkers, and the ApoE genetic profile: a longitudinal MRI study. Neurobiol Aging, 31-8, 2010.

N. Tzourio-Mazoyer, B. Landeau, D. Papathanassiou, F. Crivello, O. Etard, N. Delcroix, B. Mazoyer, , and M. Joliot. Automated Anatomical Labeling of Activations in SPM Using a Macroscopic Anatomical Parcellation of the MNI MRI Single-Subject Brain. NeuroImage, 15:273-289, 2001.

E. Yang, M. Farnum, V. Lobanov, T. Schultz, N. Raghavan, M.N. Samtani, G. Novak, V. Narayan, and A. Dibernardo. Quantifying the pathophysiological timeline of Alzheimer's disease. Journal of Alzheimer's Disease, 26(4): 745-753, 2011.

Y. Zhang, M. Brady, and S.M. Smith. Segmentation of brain mr images through 
a hidden markov random field model and the expectation maximization algorithm. IEEE Trans. Med. Imaging, 20(1):45-57, 2001. 


\section{A Implementation of the Hierarchical Model of Normal Aging}

This section describes the framework for the estimation of the group-wise longitudinal model of normal aging.

Follow-up longitudinal images were first rigidly aligned to the baseline one. The baseline image was then affine aligned to a previously defined anatomical template, which was estimated from the healthy control population under study. Follow-up images were resampled in the template space by composition of the estimated rigid and affine transformations. For each subject $S$ and time point $t$, the follow-up image $I_{t}^{S}$ was non-linearly registered to the aligned baseline $I_{0}^{S}$ with the LCC-Demons algorithm [Lorenzi et al., 2013] (Figure 3A). The LCCDemons estimates non-linear transformations parameterized by SVFs (indicated here as $\left.\exp \left(v_{t}^{S}\right)\right)$, such that $I_{0}^{S} \simeq I_{t}^{S} \circ \exp \left(v_{t}^{S}\right)$. The longitudinal morphological differences between baseline and follow-up images are in this way encoded by the series of spatially dense SVFs $v_{t}^{S}$ (Figure 1).

For each subject, a trajectory $\mu^{S}$ of the longitudinal morphological progression was estimated from the series of SVFs with a model linear in $\operatorname{time}^{2}$ (Figure $3 \mathrm{~B})$. The trajectory $\mu^{S}$ represents the estimated longitudinal morphological changes associated to a given patient $S$, and it is identified by a stationary velocity field defined voxel-wise in the anatomical space of the patient.

The direct voxel-by-voxel group-wise comparison of the trajectories is not possible, since they must be normalized in a common reference template space $T$. To normalize the trajectories $\mu^{S}$, we parallel transported them in the reference space of the anatomical template through the subject-to-template deformation.

\footnotetext{
${ }^{2}$ Note that the model is linear in the SVF parameters, but non-linear in the deformation space. In particular it always remains diffeomorphic, i.e. one-to-one and spatially differentiable (smooth). The linear model was estimated by minimizing the classical least squared criterion $\sum_{t}\left\|t \mu^{S}-v_{t}^{S}\right\|^{2}$.
} 
The parallel transport is a mathematical tool used in the context of diffeomor-

phic registration for resampling a given velocity field in the template geometry [Lorenzi and Pennec, 2013].

We therefore define the longitudinal model of normal aging in the template space $T$ (fixed effect of the trajectory) as the average trajectory $\mu_{0}$ of the transported subject-specific trajectories $\mu^{S}$ (Figure 3C).

\section{B Supplementary Table and Figures}

\begin{tabular}{|cccc|}
\hline & Healthy & MCI & AD \\
\hline \hline $\begin{array}{c}\text { Age } \\
\text { at baseline }\end{array}$ & $\mathbf{0 . 4}(<\mathbf{0 . 0 0 1})$ & $\mathbf{0 . 4 3}(<\mathbf{0 . 0 0 1})$ & $\mathbf{0 . 4 5}(<\mathbf{0 . 0 0 1})$ \\
\hline Education & $\mathbf{0 . 2 9}(\mathbf{0 . 0 0 6})$ & $0.06(0.34)$ & $0.08(0.33)$ \\
\hline $\begin{array}{c}\text { Gender } \\
(\text { Male= } 1, \text { Female }=0)\end{array}$ & $\mathbf{0 . 1 7}(\mathbf{0 . 0 9})$ & $\mathbf{0 . 3 1}(<\mathbf{0 . 0 0 1})$ & $\mathbf{0 . 2 6}(\mathbf{0 . 0 0 1})$ \\
\hline MMSE & $0.09(0.38)$ & $-0.08(0.22)$ & $-\mathbf{0 . 1 7}(\mathbf{0 . 0 3})$ \\
\hline $\begin{array}{c}\text { ADAS-cog } \\
\text { (modified) }\end{array}$ & $0.14(0.17)$ & $\mathbf{0 . 1 5}(\mathbf{0 . 0 3})$ & $\mathbf{0 . 1 4}(\mathbf{0 . 0 9})$ \\
\hline $\begin{array}{c}\text { APOE4 } \\
\text { (\# alleles) }\end{array}$ & $-0.06(0.55)$ & $-0.11(0.12)$ & $-0.13(0.1)$ \\
\hline
\end{tabular}

Table S1: Group-wise Pearson's correlation between sociodemographical/clinical scores and morphological age shift in clinical groups (p-values on parenthesis, bold for significant correlation).

\begin{tabular}{|ccccc|}
\hline & Healthy $\mathrm{A} \beta-$ & Healthy $\mathrm{A} \beta+$ & MCI stable & MCI converters \\
\hline \hline $\begin{array}{c}\text { Age } \\
\text { at baseline }\end{array}$ & $\mathbf{0 . 4}(\mathbf{0 . 0 0 3})$ & $\mathbf{0 . 3 9}(\mathbf{0 . 0 1 6})$ & $\mathbf{0 . 5 3}(<\mathbf{0 . 0 0 1})$ & $\mathbf{0 . 3 4}(<\mathbf{0 . 0 0 1})$ \\
\hline Education & $\mathbf{0 . 3 7}(\mathbf{0 . 0 0 7})$ & $0.17(0.28)$ & $-0.009(0.92)$ & $0.11(0.23)$ \\
\hline $\begin{array}{c}\text { Gender } \\
\text { Male= } 1, \text { Female }=0)\end{array}$ & $\mathbf{0 . 2 5}(\mathbf{0 . 0 7})$ & $0.04(0.77)$ & $\mathbf{0 . 3 4}(<\mathbf{0 . 0 0 1})$ & $\mathbf{0 . 2 7}(\mathbf{0 . 0 0 4})$ \\
\hline MMSE & $0.18(0.2)$ & $-0.08(0.6)$ & $-0.15(0.13)$ & $0.03(0.7)$ \\
\hline $\begin{array}{c}\text { ADAS-cog } \\
\text { (modified) }\end{array}$ & $0.12(0.39)$ & $0.16(0.33)$ & $\mathbf{0 . 2 2}(\mathbf{0 . 0 3})$ & $0.03(0.7)$ \\
\hline $\begin{array}{c}\text { APOE4 } \\
\text { \# alleles })\end{array}$ & $\mathbf{- 0 . 2 6}(\mathbf{0 . 0 5})$ & $0.009(0.95)$ & $\mathbf{- 0 . 1 8}(\mathbf{0 . 0 6})$ & $0.08(0.37)$ \\
\hline
\end{tabular}

Table S2: Group-wise Pearson's correlation between sociodemographical/clinical scores and morphological age shift in clinical subgroups (p-values on parenthesis, bold for significant correlation). 


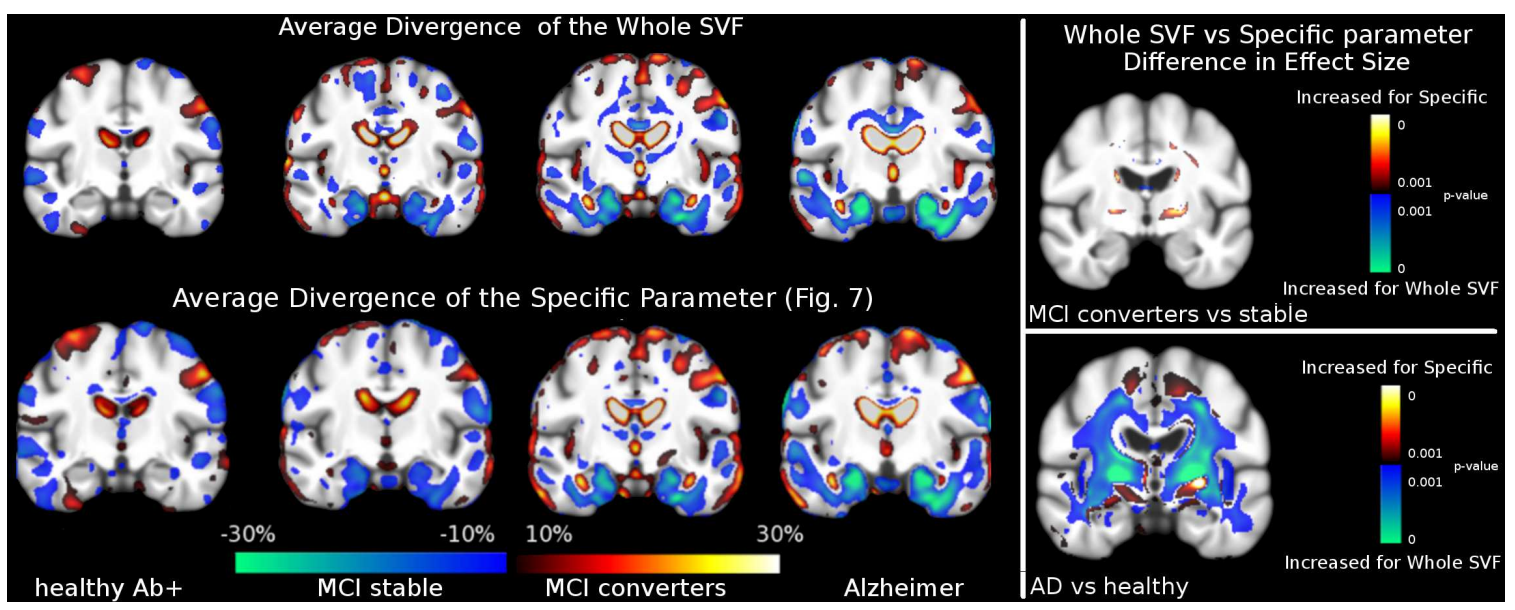

Figure S7: Left: Group-wise average divergence maps associated to the full SVFs (top), and to the specific deformation parameters (bottom). The patterns of volume change are very similar, indicating that the removal of the normal deformation parameter does not prevent the estimation of relatively larger volume changes due to specific pathological effects. Right: Statistical analysis for the differences between absolute values of the effect size obtained by using the specific parameters and the full SVFs (measured as $\frac{\left|e_{\text {whole }}\right|-\left|e s_{\text {specific }}\right|}{\text { pooled sd }}, \mathrm{p}<0.001$ uncorrected for multiple comparisons). The effect size associated to the specific deformation parameter is slightly higher around ventricles and in the white matter when comparing MCI converters with stable. When comparing AD and healthy, the whole SVF (aging+specific) provides generally higher effects size in the white matter, but slightly lower in the frontal cortex, and in the temporal horns of hippocampi. 